\title{
commodities
}

\section{Export-Led Growth}

Export of mineral commodities and economic growth: an analysis of the Export-Led Growth hypothesis for Brazil

modities

com-
boom
commodities
commodities
Export-Led Growth
$\begin{array}{ccc}- & \text { boom } \mathrm{com}- \\ \text { modities } & & \end{array}$
Commodities
Export-Led
Growth 
Led Growth

Export-

spillovers

commodities

boom

commodities

commodities

Export-Led Growth

commodities

Export-Led Growth

commodities

Export-Led Growth

commodities

- Vector Error Correction

Model

boom

commodities

commodities 


\title{
Export-Led Growth
}

\author{
Export-Led Growth
}

spillovers 
beggar thy neighbor

beggar thy neighbor

commodities

commodities

Export-Led Growth

Growth-Led Export

Export-Led Growth

\section{Export-Led Growth}

Export-Led

Growth

Export-Led Growth

Growth-Led Export 
commodities

Export-Led Growth

Export-Led Growth

Growth-Led Export

Export-Led Growth

Growth

Export-Led 
boom commodities

\section{commodities}

commodities

quantum

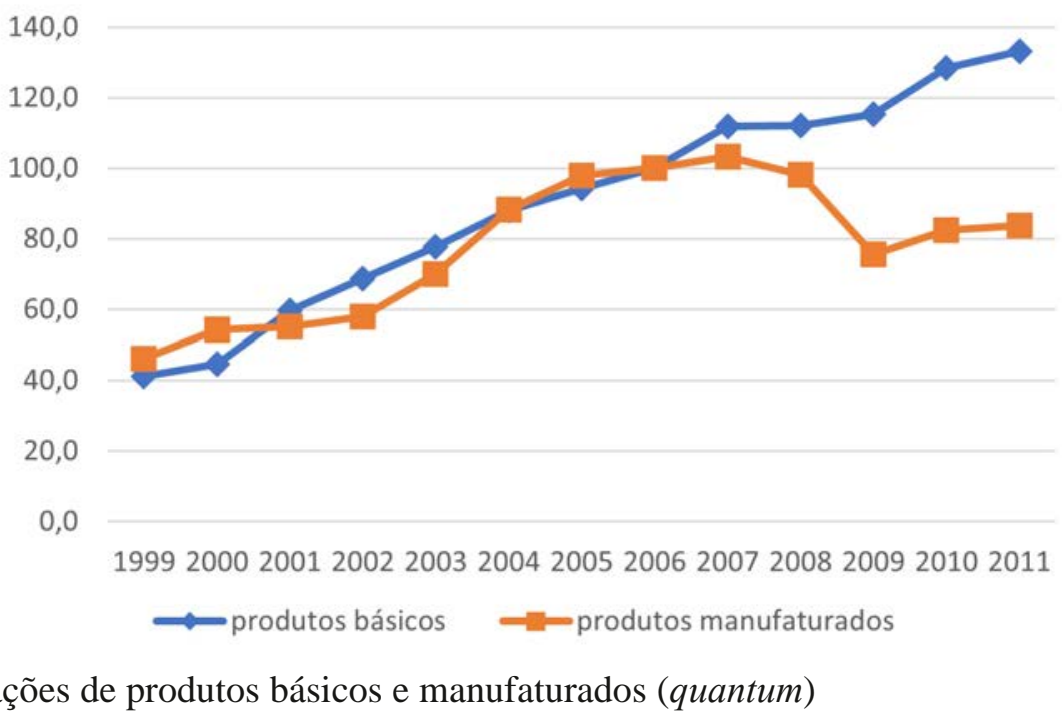




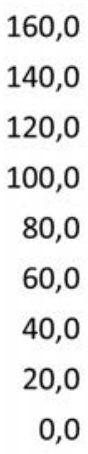

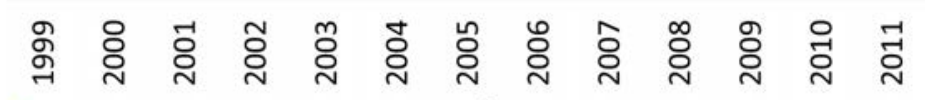

—Minerais Metálicos Quantum (Índice média $2006=100$ )

_-Alimenticios Quantum (Índice média 2006 = 100)

quantum

subprime

boom commodities

Led Growth 


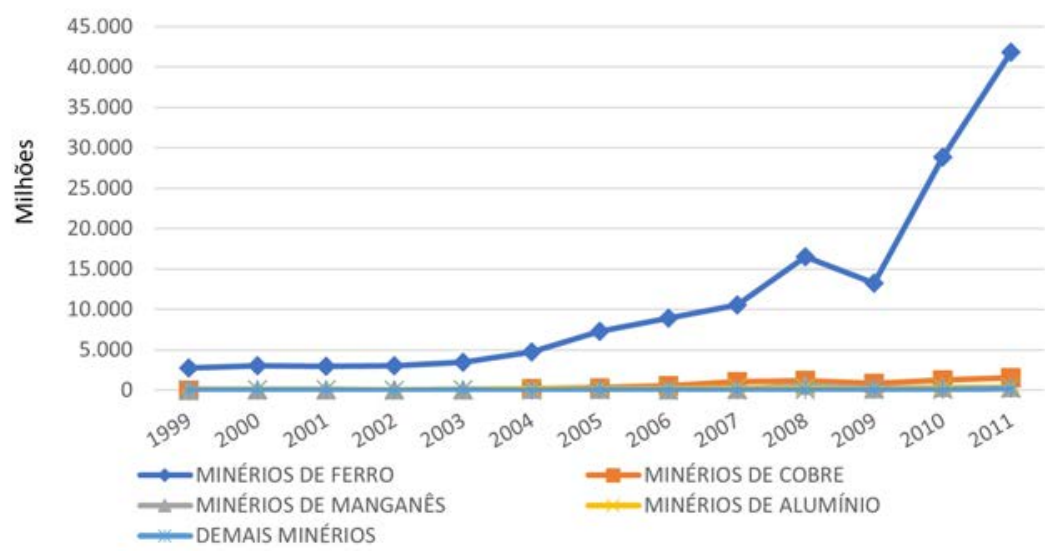

a priori

$x_{t}$ 


$$
\begin{aligned}
& \text { proxy } \\
& \text { hiato }_{t}=\sum_{i=1}^{n} \alpha_{1 i} \text { hiato }_{t-i}+\sum_{i=1}^{n} \beta_{1 i} \text { commin }_{t-i}+\sum_{i=1}^{n} \gamma_{1 i} \text { cambio }_{t-i}+\varepsilon_{1 t} \\
& \alpha \beta \gamma \\
& \text { hiato }
\end{aligned}
$$

commodities

$$
\text { Export-Led Growth }
$$

\section{commodities}

$\varepsilon$

$$
\begin{gathered}
\text { hiato }_{t}=\sum_{i=1}^{n} \alpha_{1 i} \text { hiato }_{t-i}+\sum_{i=1}^{n} \beta_{1 i} \text { alimentos }_{t-i}+\sum_{i=1}^{n} \gamma_{1 i} \text { cambio }_{t-i}+\varepsilon_{1 t} \\
\text { hiato }_{t}=\sum_{i=1}^{n} \alpha_{1 i} \text { hiato }_{t-i}+\sum_{i=1}^{n} \beta_{1 i} \text { manufaturados }_{t-i}+\sum_{i=1}^{n} \gamma_{1 i} \text { cambiot }_{t-i}+\varepsilon_{1 t} \\
\text { hiato cambio } \\
\text { manufaturados } \\
\text { alimentos } \\
\text { quantum }
\end{gathered}
$$$$
\text { quantum }
$$

\section{quantum}

cambio \\ commin}




\begin{tabular}{|c|c|c|}
\hline & hiato & \\
\hline Commodities & commin & \\
\hline & cambio & \\
\hline quantum & alimentos & \\
\hline quantum & manufaturados & \\
\hline
\end{tabular}

\begin{tabular}{ccc}
\hline & $p-$ & \\
\hline \multirow{3}{*}{ Commodities } & lag \\
& & lag \\
& & lag \\
& & lag \\
& & lag \\
\hline & & $p-$
\end{tabular}

commodities 
$p$ -

\begin{tabular}{ccc} 
& & \\
\hline \multirow{3}{*}{ Commodities } & lag \\
& & $\operatorname{lag}$ \\
& & $\operatorname{lag}$ \\
& & $\operatorname{lag}$ \\
& & $\operatorname{lag}$ \\
\hline- & $p-$ & $p-$
\end{tabular}

$\lambda_{\text {trace }} p-$ $\lambda_{\max } p-$

p- 
$\lambda_{\text {trace }} p$ -

$\lambda_{\max } p-$

p-

commodities

boom

commodities

quantum

boom commodities 
quantum

boom commodities 
commodities

Z $\quad P \quad z$

$-$

$P \quad z$

commodities

commodities

boom commodities

commodities

spillovers 


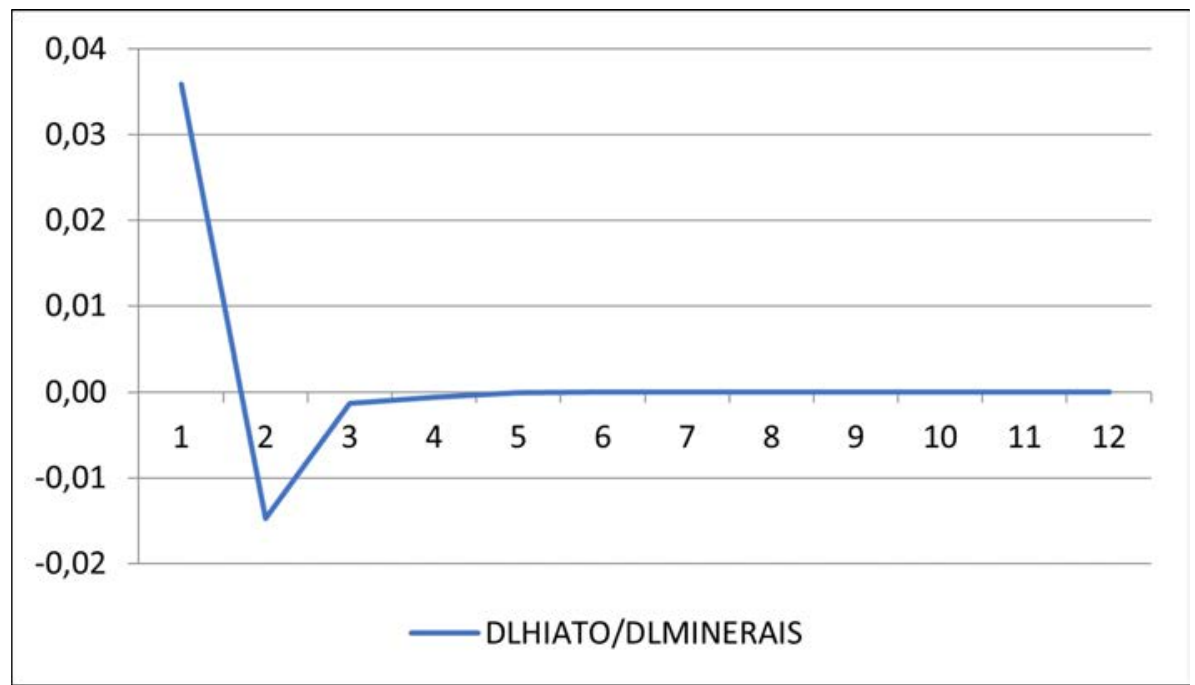

commodities

boom commodities

commodities

commodities 


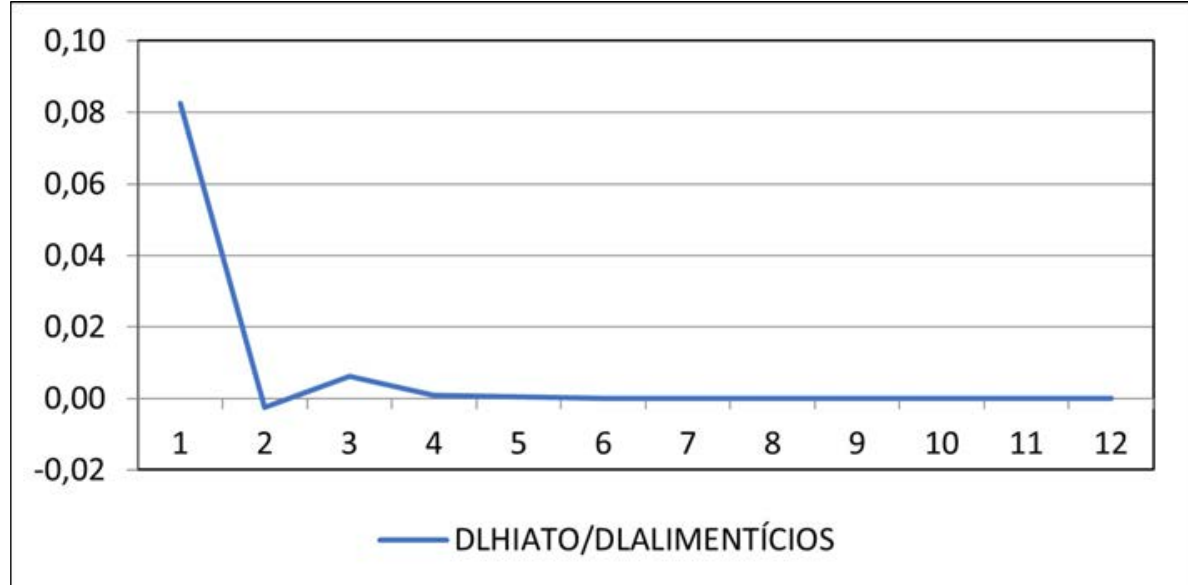


boom commodities 


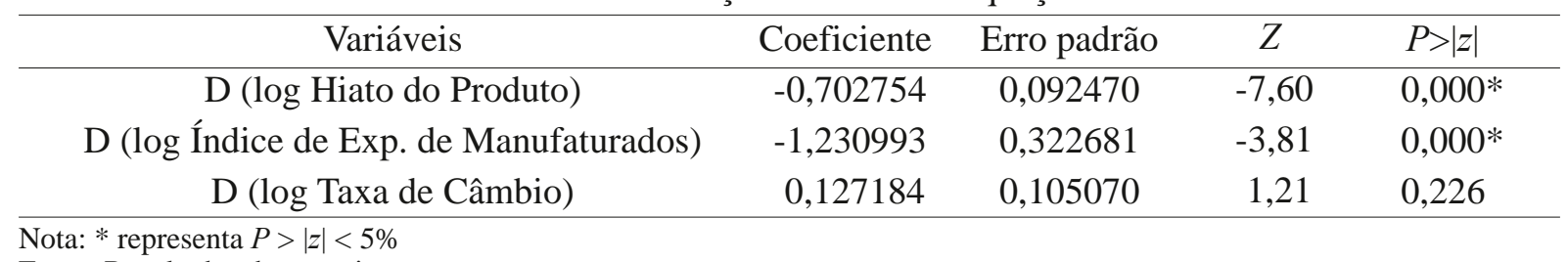

commodities

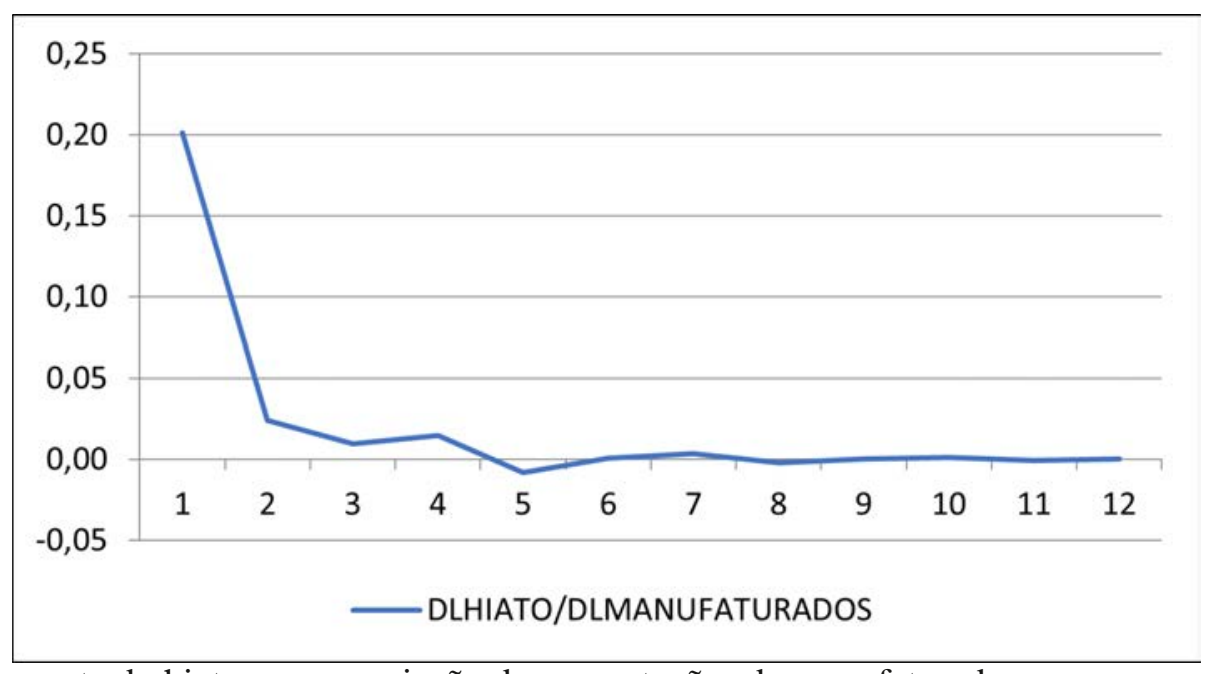

boom commodities 
commodities

boom commodities

commodities

commodities

commodities

commodities 
boom commodities

proxy

commodities

\section{proxy}

commodities

commodities

commodities

commodities

commodities

Export-Led Growth 


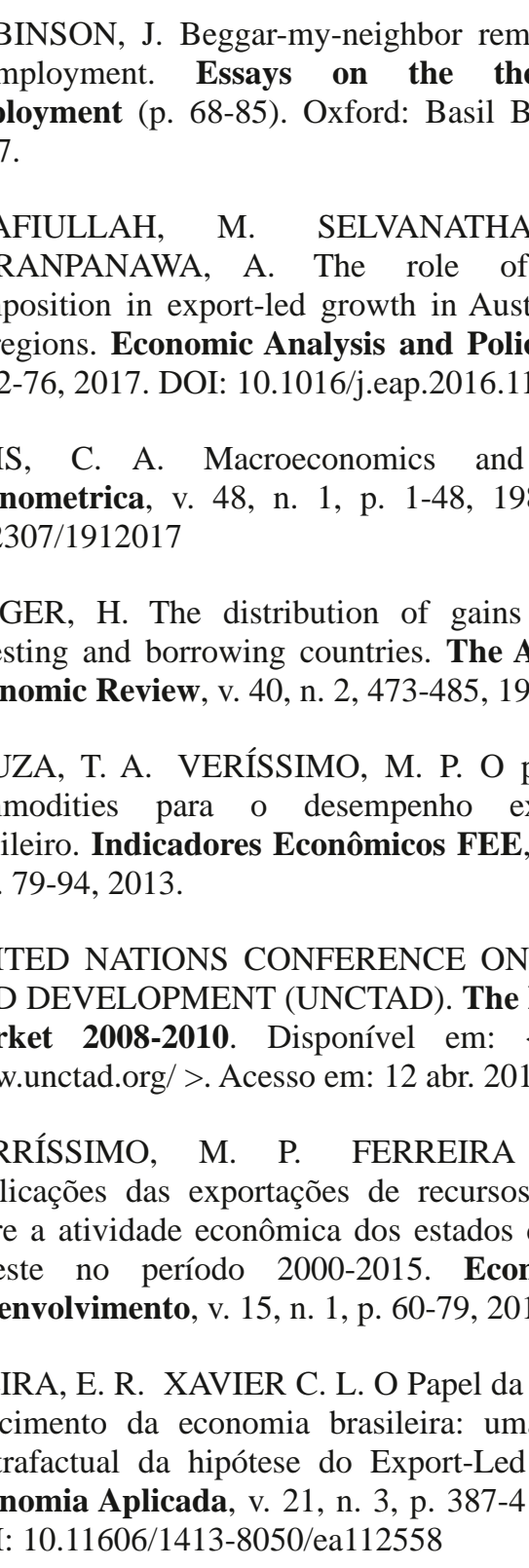

